\title{
Atrial fibrillation and rapid atrial pacing are linked to enhanced inflammation and altered fibrinolysis in patients undergoing pulmonary vein isolation
}

\author{
Maciej T. Wybraniec ${ }^{1}$, Joanna Wieczorek ${ }^{1}$, Iwona Woźniak-Skowerska ${ }^{1}$, Seweryn Nowak ${ }^{1}$, \\ Anna Wnuk-Wojnar ${ }^{1}$, Anetta Undas² ${ }^{2}$ Katarzyna Mizia-Stec ${ }^{1}$ \\ ${ }^{1} 1^{\text {st }}$ Department of Cardiology, School of Medicine in Katowice, Medical University of Silesia, Katowice, Poland \\ ${ }^{2}$ Institute of Cardiology, Jagiellonian University Medical College, Krakow Centre for Medical Research and Technology, \\ John Paul II Hospital, Krakow, Poland
}

\section{INTRODUCTION}

Atrial fibrillation (AF) increases the risk of cardio-embolic stroke [1]. Patients with AF in sinus rhythm (SR) are also characterised by a hypercoagulable state [2], which is further augmented by a coexisting inflammatory response [3]. The prothrombotic state in AF is associated with increased thrombin formation, higher von Willebrand factor (vWF), and increased plasminogen activator inhibitor-1 (PAI-1), with altered fibrin clot properties [2, 3]. Hypercoagulability has been shown to be linked with left atrial (LA) remodelling and wall stress [4]. In patients without documented AF, the LA appendage thrombus was associated with prothrombotic blood alterations [5]. Elevated levels of proinflammatory interleukin 6 (II-6) and C-reactive protein were associated with increased thromboembolic risk in AF patients [3].

Previous studies have provided conflicting evidence regarding haemostasis and inflammatory status in response to rapid atrial pacing $[6,7]$. It is likely that a hypercoagulable state and inflammation may be at least partially dependent on the tachyarrhythmia that is associated with $\mathrm{AF}$, emphasising the importance of rate control [6].

Pulmonary vein isolation (PVI) is a beneficial procedure to manage the symptoms in patients with AF [8]. It has been documented that the LA scarring associated with PVI increases the risk of cerebral embolism [9]. The mechanism of this association has not been fully elucidated.

We sought to evaluate the LA and peripheral haemostasis, fibrinolysis, and inflammatory response in patients with AF during a PVI procedure and following an episode of rapid atrial pacing.

\section{METHODS}

A total of 14 patients with either paroxysmal or persistent $A F$, referred for $\mathrm{PVI}$, were enrolled in this preliminary, prospective, observational study. All patients gave their written, informed consent to participate. The study was approved by the local Ethics Committee and complied with the principles of the Declaration of Helsinki. The exclusion criteria were lack of adequate anticoagulation in the month preceding the procedure, prior ischaemic or haemorrhagic stroke, venous or arterial thromboembolism, estimated glomerular filtration rate $<60 \mathrm{~mL} / \mathrm{min} / 1.73 \mathrm{~m}^{2}$, pregnancy, and age $<18$ and $>80$ years.

Blood samples were obtained at the beginning of the $\mathrm{PVI}$ procedure (from the peripheral vein [PV]), and during the PVI procedure: LA1 — immediately after the interatrial septum puncture, prior to heparin administration; LA2 - at least $5 \mathrm{~min}$ after the administration of an unfractionated heparin IV bolus $(100 \mathrm{IU} / \mathrm{kg}$ ) with activated clotting time (ACT) of $>300 \mathrm{~s}$, before the application of radiofrequency (RF) energy; and LA3 - after successful isolation of all pulmonary veins. The patients who were in SR underwent rapid atrial pacing at the rate of $150 \mathrm{bpm}$ prior to the application of RF energy. The interval between rapid atrial pacing and the ablation was $20 \mathrm{~min}$.

We determined plasma vWF using a latex immunoassay (Diagnostica Stago, Asnières, France), whereas soluble P-selectin and II-6 (R\&D Systems, Minneapolis, MN, USA) as well as tissue plasminogen activator (tPA) antigen and PAI-1 antigen (American Diagnostica, Stamford, CT, USA) were determined using enzyme-linked immunosorbent assays (ELISA).

\section{Address for correspondence:}

Maciej T. Wybraniec, MD, PhD, $1^{\text {st }}$ Department of Cardiology, School of Medicine in Katowice, Medical University of Silesia, ul. Ziołowa 47, 40-635 Katowice, Poland, tel: +48 3235988 90, fax: +48 3225230 32, e-mail: maciejwybraniec@gmail.com

Received: 11.12.2018 Accepted: 28.12.2018 Available as AoP: 28.12.2018

Kardiologia Polska Copyright @ Polish Cardiac Society 2019 
Table 1. Comparison of the biomarker concentrations in the left atrium at different time points during ablation

\begin{tabular}{|c|c|c|c|c|c|c|c|}
\hline \multirow[t]{2}{*}{ Variables } & \multirow[t]{2}{*}{ LA1* } & \multirow[t]{2}{*}{ LA2* } & \multirow[t]{2}{*}{ LA3* } & \multicolumn{3}{|c|}{$\mathrm{P}^{* *}$} & \multirow[t]{2}{*}{$P^{* * *}$} \\
\hline & & & & LA1 vs. LA2 & LA1 vs. LA3 & LA2 vs. LA3 & \\
\hline vWF [IU/dL] & $198.0(188.0-210.0)$ & 209.5 (199.0-220.0) & $200.5(187.0-212.0)$ & 0.490 & 0.778 & 0.300 & 0.395 \\
\hline $\mathrm{sPs}[\mathrm{ng} / \mathrm{mL}]$ & $100.5(94.0-111.0)$ & $106.0(98.0-112.0)$ & $91.5(83.0-111.0)$ & 0.490 & 0.152 & 0.149 & 0.458 \\
\hline IL-6 [pg/mL] & $11.3(8.6-11.9)$ & $12.7(10.5-13.9)$ & $14.2(12.0-15.4)$ & 0.011 & 0.001 & 0.001 & 0.0001 \\
\hline $\mathrm{tPA}[\mathrm{ng} / \mathrm{mL}]$ & $6.81(5.81-7.98)$ & $6.99(5.87-8.06)$ & $11.3(10.3-13.3)$ & 0.826 & 0.001 & 0.001 & $<0.0001$ \\
\hline PAI-1 [ng/mL] & $15.1(12.0-17.3)$ & $14.2(11.5-16.0)$ & $16.5(15.6-18.5)$ & 0.754 & 0.084 & 0.011 & 0.008 \\
\hline
\end{tabular}

*Data are presented as median and one and three quartile boundaries (1-3Q); **Wilcoxon matched pairs test; ***Friedman analysis of variance (ANOVA); II-6 - interleukin 6; LA1 - blood withdrawn immediately after the interatrial septum puncture, prior to heparin administration; LA2 - blood withdrawn $\geq 5$ min or after the administration of an unfractionated heparin IV bolus (100 IU/kg) with activated clotting time of $>300 \mathrm{~s}$, before application of radiofrequency energy; LA3 - blood withdrawn after successful isolation of all pulmonary veins; in patients in sinus rhythm at baseline, the ablation was preceded by a 5-min episode of rapid atrial pacing at 150 bpm; PAI-1 — plasminogen activator inhibitor-1; sPs — soluble P-selectin; tPA — tissue plasminogen activator antigen; vWF — von Willebrand factor

All patients received vitamin $\mathrm{K}$ antagonists (VKA) for at least one month prior to PVI. PVI was preceded by transoesophageal echocardiography within $24 \mathrm{~h}$ before the procedure. LA rotational angiography was performed at the beginning of the procedure. Following the transseptal puncture, three-dimensional electro-anatomical mapping was performed using the CARTO ${ }^{\circledR}$ system (Biosense Webster, Diamond Bar, CA, USA) in patients undergoing RF ablation with a ThermoCool ${ }^{\circledR}$ SmartTouch ${ }^{\circledR}$ SF catheter (Biosense Webster).

In patients who were in SR during the procedure, the ablation was preceded by a 5-min episode of rapid atrial pacing at $150 \mathrm{bpm}$ in order to compensate for the lack of arrhythmia. The interval between pacing and PVI in these patients was 20 min.

The procedure was performed with an international normalised ratio (INR) of up to 2.5 (preferably 2.0-2.5). All patients received unfractionated heparin (IV bolus $100 \mathrm{IU} / \mathrm{kg}$ ) immediately after the transseptal puncture and a continuous infusion of $2000 \mathrm{IU} / \mathrm{h}$ through the transseptal sheath to obtain an ACT over $300 \mathrm{~s}$, measured at 30-min intervals during the procedure. A postprocedural 24-h heparin infusion under ACT control was continued in patients with an INR $<2.0$ during the procedure. The next VKA dose was administered $4 \mathrm{~h}$ after the procedure to obtain an INR within the range of 2.0-3.0 on the following day.

\section{Statistical analysis}

The statistical analysis was performed using the MedCalc v.14.8.1 (MedCalc Software, Ostend, Belgium). Distribution of variables was verified using the Shapiro-Wilk test. Temporal variations of the biomarker concentration were evaluated using Wilcoxon matched pairs test for dependent samples and Friedman analysis of variance (ANOVA). A p-value $<0.05$ was considered statistically significant.

\section{RESULTS AND DISCUSSION}

We studied 14 symptomatic AF patients at a mean age of 57.5 (43-64) years (men 57.1\%) with a high prevalence of arterial hypertension ( $\mathrm{n}=12,85.7 \%)$, hyperlipidaemia $(n=10,71.4 \%)$, and coronary artery disease $(n=4,28.6 \%)$. All patients had a normal left ventricular ejection fraction (LVEF: 55\%, interquartile range: 50\%-60\%). Paroxysmal AF was diagnosed in six (42.9\%) patients, and PVI was performed in this sub-group while the patients remained in SR. The $\mathrm{CHA}_{2} \mathrm{DS}_{2}$-VASc score was 2 (1-3) points.

Tissue plasminogen activator antigen concentration was higher (6.81 [5.81-7.98] vs. 5.76 [4.76-6.71] ng/mL, $\mathrm{p}=0.002$, respectively), while PAI-1 levels tended to be higher (15.1 [12.0-17.3] vs. 13.0 [10.3-16.5] ng/mL, $p=0.090$, respectively) in the LA than in the PV. Plasma vWF $(p=0.23)$, soluble P-selectin $(p=1.00)$, and II-6 $(p=0.157)$ were similar in the venous and LA samples.

A serial biomarker sampling during the procedure revealed that the II-6 levels gradually increased after the transseptal puncture (LA1 vs. LA2) and even more (LA2 vs. LA3) after PVI (Table 1). Although tPA and PAI-1 levels did not change significantly after the transseptal puncture, AF ablation led to a marked increase in both biomarkers, while vWF and soluble P-selectin concentrations remained unchanged in all samples (Table 1).

Patients who had had PVI performed in AF were characterised by higher tPA in LA (LA2: 7.9 [6.9-8.7] vs. 5.8 [5.7-6.7] $\mathrm{ng} / \mathrm{mL}, \mathrm{p}=0.008$ ) and a lower vWF concentration (LA2: 200.0 [191.0-207.5] vs. 219.5 [213.0-236.0] IU/dL, $\mathrm{p}=0.014)$ compared to those in SR before PVI. There were no differences in terms of PAI-1 levels at LA1 $(p=0.401)$ and LA2 ( $p=0.175)$, but PAI-1 level at LA3 tended to be higher in patients ablated in AF compared with those in SR (17.9 [16.4-20.4] vs. 15.6 [14.9-16.2] ng/mL, $p=0.081$ ). II-6 remained comparable between the preprocedural SR and AF groups.

The present study suggests enhanced inflammation secondary to mechanical manipulation during transseptal puncture, which was further potentiated by application of RF energy, and impaired fibrinolysis secondary to ablation. 
Although the presence of AF during the procedure is associated with increased tPA antigen, it does not lead to platelet activation, reflected by soluble P-selectin or the inflammatory response as shown by II-6. The relative increase of tPA and decrease of $\mathrm{VWF}$ antigen, both of which are mainly released from endothelial cells, may reflect complex alterations to markers of endothelial injury during an active AF episode.

Our results are in line with findings in an animal model, suggesting that local inflammation, reflected by tumour necrosis factor alpha (TNF- $\alpha$ ) and II- 6 , might be induced by a prolonged rapid atrial pacing [7]. Although Lim et al. [6] corroborated that both $\mathrm{AF}$ and rapid atrial pacing at $150 \mathrm{bpm}$ led to LA platelet activation (P-selectin elevation) and increased thrombin generation, previous reports did not show elevated PAI-1 antigen following an episode of induced high atrial rate, suggesting PAI-1-driven impaired fibrinolysis in this setting.

The major study limitation is the LA3 sample acquisition, because changes in II- 6 and PAI- 1 could be triggered by both RF ablation and rapid atrial pacing in patients who were in SR at baseline. The study represented a preliminary report of a larger investigation, in which blood was acquired from LA at four time points, including an additional LA blood acquisition after rapid atrial pacing and prior to ablation. Unfortunately, in this study we measured biomarkers only at three time points, hence at LA3 the biomarkers were measured after ablation (persistent AF) or after rapid atrial pacing and subsequent ablation (paroxysmal AF patients only with SR at the beginning of the procedure). Presumably, rapid atrial pacing performed 20 min before the initiation of ablation had a minor impact on PAI-1 decrease and II-6 increase, which was primarily mediated by ablation-related endocardial injury. Ideally, two sample acquisitions: (1) after rapid atrial pacing, and (2) after application of RF energy should be performed. Still, these data lay the foundation for the notion that PVI is related to inflammation and impaired fibrinolysis, presumably translating into increased risk of periprocedural thrombotic complications.

In conclusion, the current preliminary results suggest that initial mechanical LA manipulation and PVI itself preceded by rapid atrial pacing are associated with a pronounced inflammatory response, while RF ablation and rapid atrial pacing seem to be associated with impaired fibrinolysis. Larger studies are needed to confirm these observations.
Funding: The study was supported by a grant from the Medical University of Silesia (grant No. KNW-1-115/N/8/K to K.M.-S.), and a grant from Jagiellonian University Medical College (grant No. K/ZDS/007717 to A.U.).

\section{Conflict of interest: none declared}

\section{References}

1. Wolf PA, Abbott RD, Kannel WB. Atrial fibrillation as an independent risk factor for stroke: the Framingham Study. Stroke. 1991; 22(8): 983-988, indexed in Pubmed: 1866765.

2. Drabik L, Wołkow P, Undas A. Denser plasma clot formation and impaired fibrinolysis in paroxysmal and persistent atrial fibrillation while on sinus rhythm: association with thrombin generation, endothelial injury and platelet activation. Thromb Res. 2015; 136(2): 408-414, doi : 10.1016/j.thromres.2015.05.028, indexed in Pubmed: 26048399.

3. Aulin J, Siegbahn A, Hijazi Z, et al. Interleukin-6 and C-reactive protein and risk for death and cardiovascular events in patients with atrial fibrillation. Am Heart J. 2015; 170(6): 1151-1160, doi: 10.1016/j.ahj.2015.09.018.

4. Matusik PT, Matusik PS, Kornacewicz-Jach Z, et al. Elevated NT-proBNP is associated with unfavorably altered plasma fibrin clot properties in atrial fibrillation. Int J Cardiol. 2017; 243: 244-250, doi: 10.1016/j.ijcard.2017.05.060, indexed in Pubmed: 28571619.

5. Meus R, Son M, Sobczyk D, et al. Prothrombotic state in patients with a left atrial appendage thrombus of unknown origin and cerebrovascular events. Stroke. 2016; 47(7): 1872-1878, doi: 10.1161/STROKEAHA.116.012856, indexed in Pubmed: 27301942.

6. Lim HS, Willoughby SR, Schultz C, et al. Effect of atrial fibrillation on atrial thrombogenesis in humans: impact of rate and rhythm. J Am Coll Cardiol. 2013; 61(8): 852-860, doi: 10.1016/j. jacc.2012.11.046, indexed in Pubmed: 23333141.

7. Zhao Q, Zhang S, Zhao H, et al. Median nerve stimulation prevents atrial electrical remodelling and inflammation in a canine model with rapid atrial pacing. Europace. 2018; 20(4): 712-718, doi: 10.1093/europace/eux003, indexed in Pubmed: 28379329.

8. Wynn GJ, Das M, Bonnett LJ, et al. Efficacy of catheter ablation for persistent atrial fibrillation: a systematic review and meta-analysis of evidence from randomized and nonrandomized controlled trials. Circ Arrhythm Electrophysiol. 2014; 7(5): 841-852, doi: 10.1161/CIRCEP.114.001759, indexed in Pubmed: 25132078.

9. Larbig R, Dittrich R, Kochhaeuser S, et al. Influence of clinical parameters and anticoagulation on intraprocedural cerebral microembolic signals during pulmonary vein isolation. PLoS One. 2016; 11(6): e0157886, doi: 10.1371/journal.pone.0157886, indexed in Pubmed: 27327662.

Cite this article as: Wybraniec MT, Wieczorek J, Woźniak-Skowerska I, et al. Atrial fibrillation and rapid atrial pacing are linked to enhanced inflammation and altered fibrinolysis in patients undergoing pulmonary vein isolation. Kardiol Pol. 2019; 77(3): 386-388, doi: 10.5603/KP.a2018.0246. 